\title{
Current-voltage characteristics of tunnel Josephson junctions with a ferromagnetic interlayer
}

\author{
A. S. Vasenko, ${ }^{1,2,3}$ S. Kawabata, ${ }^{4}$ A. A. Golubov, ${ }^{5}$ M. Yu. Kupriyanov, ${ }^{6}$ C. Lacroix, ${ }^{7}$ F. S. Bergeret,${ }^{3,8}$ and F. W. J. Hekking ${ }^{2}$ \\ ${ }^{1}$ Institut Laue-Langevin, 6 rue Jules Horowitz, BP 156, F-38042 Grenoble, France \\ ${ }^{2}$ LPMMC, Université Joseph Fourier and CNRS, 25 avenue des Martyrs, BP 166, F-38042 Grenoble, France \\ ${ }^{3}$ Donostia International Physics Center (DIPC), Manuel de Lardizabal 4, E-20018 San Sebastián, Spain \\ ${ }^{4}$ Nanosystem Research Institute (NRI), National Institute of Advanced Industrial Science and Technology (AIST), \\ and JST-CREST, Tsukuba, Ibaraki 305-8568, Japan \\ ${ }^{5}$ Faculty of Science and Technology and MESA ${ }^{+}$Institute for Nanotechnology, University of Twente, NL-7500 AE Enschede, The Netherlands \\ ${ }^{6}$ Nuclear Physics Institute, Moscow State University, Moscow 119992, Russia \\ ${ }^{7}$ Institut Néel, Université Joseph Fourier and CNRS, 25 avenue des Martyrs, BP 166, F-38042 Grenoble, France \\ ${ }^{8}$ Centro de Física de Materiales (CFM-MPC), Centro Mixto CSIC-UPV/EHU, Manuel de Lardizabal 5, E-20018 San Sebastián, Spain
}

(Received 11 January 2011; revised manuscript received 26 May 2011; published 21 July 2011)

\begin{abstract}
We present a quantitative study of the current-voltage characteristics (CVC) of diffusive superconductor/ insulator/ferromagnet/superconductor (SIFS) tunnel Josephson junctions. In order to obtain the CVC we calculate the density of states (DOS) in the F/S bilayer for arbitrary length of the ferromagnetic layer, using quasiclassical theory. For a ferromagnetic layer thickness larger than the characteristic penetration depth of the superconducting condensate into the F layer, we find an analytical expression which agrees with the DOS obtained from a self-consistent numerical method. We discuss general properties of the DOS and its dependence on the parameters of the ferromagnetic layer. In particular we focus our analysis on the DOS oscillations at the Fermi energy. Using the numerically obtained DOS we calculate the corresponding CVC and discuss their properties. Finally, we use CVC to calculate the macroscopic quantum tunneling (MQT) escape rate for the current biased SIFS junctions by taking into account the dissipative correction due to the quasiparticle tunneling. We show that the influence of the quasiparticle dissipation on the macroscopic quantum dynamics of SIFS junctions is small, which is an advantage of SIFS junctions for superconducting qubits applications.
\end{abstract}

DOI: 10.1103/PhysRevB.84.024524

PACS number(s): 74.45.+c, 74.50.+r, 74.78.Fk, 75.30.Et

\section{INTRODUCTION}

The possibility to switch the ground state of a Josephson junction from a 0 to a $\pi$ phase state and the possible application of such junctions in quantum information led to a renewal of interest in the study of the so-called $\pi$ Josephson junctions. The existence of such a transition was predicted more than thirty years ago; ${ }^{1}$ however due to technological requirements only recently it was observed. The realization of $\pi$ Josephson junctions was achieved in superconductor/ ferromagnet/superconductor (SFS) junctions. ${ }^{2-21}$ Microscopically, S/F hybrid structures are characterized by an unusual proximity effect, with a damped oscillatory behavior of the superconducting correlations in the F layer (for a review see Refs. 22-24 and references therein). This unusual proximity effect in S/F layered structures leads to a number of striking phenomena such as the nonmonotonic dependence of their critical temperature and the appearance of oscillations of critical current in SFS Josephson junctions as a function of the F-layer thickness. ${ }^{2,9}$ In particular the change of sign of the critical current corresponds to the so-called $0-\pi$ transition.

On the other hand, SFS junctions, as any metallic junction, exhibit very small resistances and therefore are not quite suitable for those applications, for which active Josephson junctions are required. This problem can be solved by adding an additional insulating (I) layer to increase the resistance. SIFS junctions represent an interesting case for practical use of $\pi$ Josephson junctions. For instance, a SIFS structure offers the freedom to tune the critical current density over a wide range and at the same time realize high values of the product of the junction critical current $I_{c}$ and its normal state resistance $R_{n} \cdot{ }^{13-15} \mathrm{In}$ addition, $\mathrm{Nb}$ based tunnel junctions are usually underdamped, which is desired for many applications. Due to these advantages, SIFS $\pi$ junctions have been proposed as potential elements in superconducting classical and quantum logic circuits. ${ }^{25,26}$ For instance, SIFS junctions can be used as complementary elements ( $\pi$ shifters) in RSFQ circuits (see Ref. 27 and references therein). Finally, SIFS structures have been proposed for the realization of so-called $\varphi$ junctions with a $\varphi$ drop in the ground state, where $0<\varphi<\pi{ }^{28}$ The properties of SIFS junctions have been intensively studied both experimentally ${ }^{9-18}$ and theoretically. ${ }^{28-31}$ However, properties of the quasiparticle current have received relatively little attention so far, although they can be very important for the description of SIFS junctions as possible elements of superconducting logic circuits.

The purpose of this work is to provide a quantitative model describing the behavior of quasiparticle current in SIFS junctions as a function of parameters characterizing material properties of the ferromagnetic interlayer. We also focus our study on the properties of the density of states (DOS) in S/F bilayers and discuss the oscillations of the DOS at Fermi energy. Finally, we calculate the macroscopic quantum tunneling (MQT) escape rate for current-biased SIFS junctions by taking into account the dissipative correction due to the quasiparticle tunneling. Based on this we conclude that the influence of the quasiparticle dissipation on the macroscopic quantum dynamics of SIFS junctions is small, which is an advantage of SIFS junctions for quantum logic (qubit) applications. 
The paper is organized as follows. In the next section we formulate the theoretical model and the basic equations. In Sec. III we solve the nonlinear Usadel equations numerically for arbitrary length of the ferromagnetic layer, and calculate the DOS in the F layer. We compare these results with an analytical expression for DOS in case of a long SIFS junction, i.e., when the thickness $d_{f}$ of the ferromagnetic layer is much larger than the decay length of the characteristic superconducting correlations in the ferromagnet $\xi_{f 1}$. We also discuss the oscillations of the DOS at the Fermi energy. In Sec. IV we present the current-voltage characteristics of SIFS junctions for different parameters of the ferromagnetic interlayer. In Sec. V, we use these data to calculate the MQT escape rate for current-biased SIFS junctions by taking into account the dissipative effect of the quasiparticle tunneling. Finally we summarize the results in Sec. VI.

\section{MODEL AND BASIC EQUATIONS}

We consider a SIFS junction such as the one depicted in Fig. 1. It consists of a ferromagnetic layer of thickness $d_{f}$ and two thick superconducting electrodes along the $x$ direction. The left and right superconductor/ferromagnet interfaces are characterized by the dimensionless parameters $\gamma_{B 1}$ and $\gamma_{B 2},{ }^{32,33}$ respectively, where $\gamma_{B 1, B 2}=R_{B 1, B 2} \sigma_{n} / \xi_{n}, R_{B 1, B 2}$ are the resistances of the left and right $\mathrm{S} / \mathrm{F}$ interfaces, respectively, $\sigma_{n}$ is the conductivity of the F layer, $\xi_{n}=\sqrt{D_{f} / 2 \pi T_{c}}, D_{f}$ is the diffusion coefficient in the ferromagnetic metal, and $T_{c}$ is the critical temperature of the superconductor (we assume $\hbar=k_{B}=1$, except for Sec. V). We also assume that the $\mathrm{S} / \mathrm{F}$ interfaces are not magnetically active. We will consider the diffusive limit, in which the elastic scattering length $\ell$ is much smaller than the decay characteristic length $\xi_{f 1}=\min \left[\xi_{f 1 \uparrow}, \xi_{f 1 \downarrow}\right.$ ] [for the definitions of $\xi_{f 1 \uparrow(\downarrow)}$ see Eqs. (16) below].

We assume that the tunneling barrier is located at the left $\mathrm{S} / \mathrm{F}$ interface, while the right interface is perfectly transparent; this means that $\gamma_{B 1} \gg 1$ while $\gamma_{B 2} \ll 1$. In this case the left $S$ layer and the right $\mathrm{F} / \mathrm{S}$ bilayer in Fig. 1 are decoupled and we can calculate the quasiparticle current through a SIFS junction using the standard tunneling formula ${ }^{34}$

$$
I=\frac{1}{e R} \int_{-\infty}^{\infty} d E N_{s}(E-e V) N_{f}(E)[f(E-e V)-f(E)],
$$

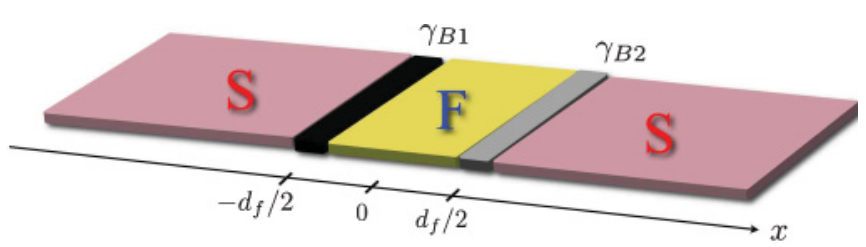

FIG. 1. (Color online) Geometry of the considered system. The thickness of the ferromagnetic interlayer is $d_{f}$. The transparency of the left (right) $\mathrm{S} / \mathrm{F}$ interface is characterized by the coefficient $\gamma_{B 1(B 2)}$. The left interface is an insulating barrier, $\gamma_{B 1} \gg 1$ (shown by a black line), while the right interface is transparent, $\gamma_{B 2} \ll 1$ (shown by a gray line). where $N_{s}(E)=|E| \Theta(|E|-\Delta) / \sqrt{E^{2}-\Delta^{2}}$ is the BCS DOS, $\Theta(x)$ is the Heaviside step function, $N_{f}(E)$ is the DOS in the ferromagnetic interlayer at $x=-d_{f} / 2, f(E)=[1+$ $\exp (E / T)]^{-1}$ is the Fermi function, and $R \equiv R_{B 1}$. Both $N_{s}(E)$ and $N_{f}(E)$ are normalized to their values in the normal state. In particular at zero temperature, $T=0$, the current acquires the form

$$
I=\Theta(e V-\Delta) \frac{1}{e R} \int_{0}^{e V-\Delta} d E N_{s}(E-e V) N_{f}(E) .
$$

To obtain $N_{f}(E)$ we notice that since $\gamma_{B 1} \gg 1$, the left superconducting lead does not influence the DOS in the ferromagnetic interlayer (to zero order in the barrier transparency). This reduces the problem to the following: We need to find the DOS of a single $\mathrm{F} / \mathrm{S}$ bilayer, which can be done by solving the Usadel equations in the ferromagnetic layer.

Using the $\theta$ parameterizations of the normal and anomalous Green's functions, $G=\cos \theta, F=\sin \theta$, we can write the Usadel equations in the $F$ layer as ${ }^{35-37}$

$$
\begin{aligned}
\frac{D_{f}}{2} \frac{\partial^{2} \theta_{f \uparrow(\downarrow)}}{\partial x^{2}}= & \left(\omega \pm i h+\frac{1}{\tau_{z}} \cos \theta_{f \uparrow(\downarrow)}\right) \sin \theta_{f \uparrow(\downarrow)} \\
& +\frac{1}{\tau_{x}} \sin \left(\theta_{f \uparrow}+\theta_{f \downarrow}\right) \pm \frac{1}{\tau_{s o}} \sin \left(\theta_{f \uparrow}-\theta_{f \downarrow}\right),
\end{aligned}
$$

where the positive and negative signs correspond to the spin-up $\uparrow$ and spin-down $\downarrow$ states, respectively. In this notation the spin-up state corresponds to the anomalous Green's function $F_{\uparrow} \sim\left\langle\psi_{\uparrow} \psi_{\downarrow}\right\rangle$ while the spin-down state corresponds to $F_{\downarrow} \sim$ $\left\langle\psi_{\downarrow} \psi_{\uparrow}\right\rangle$, where $\psi_{\uparrow(\downarrow)}$ are the electron fermionic operators. The $\omega=2 \pi T\left(n+\frac{1}{2}\right)$ are the Matsubara frequencies, and $h$ is the exchange field in the ferromagnet. The scattering times are labeled here as $\tau_{z}, \tau_{x}$, and $\tau_{s o}$, where $\tau_{z(x)}$ corresponds to the magnetic scattering parallel (perpendicular) to the quantization axis, and $\tau_{s o}$ is the spin-orbit scattering time. ${ }^{38-41}$

We consider here ferromagnets with a strong uniaxial anisotropy, in which case the magnetic scattering does not couple the spin-up and spin-down electron populations; i.e., the perpendicular fluctuations of the exchange field are suppressed $\left(\tau_{x}^{-1} \sim 0\right)$. Therefore, we will neglect $\tau_{x}$ in our consideration and denote $\tau_{z}$ as a magnetic scattering time $\tau_{m}$. We will also consider ferromagnets with weak spin-orbit interactions and henceforth also neglect the spin-orbit scattering time $\tau_{s o}$. In this case the Usadel equations in the ferromagnetic layer for different spin projections are not coupled any more and can be written as

$$
\frac{D_{f}}{2} \frac{\partial^{2} \theta_{f \uparrow(\downarrow)}}{\partial x^{2}}=\left(\omega \pm i h+\frac{\cos \theta_{f \uparrow(\downarrow)}}{\tau_{m}}\right) \sin \theta_{f \uparrow(\downarrow)},
$$

while in the $\mathrm{S}$ layer the Usadel equations take the form

$$
\frac{D_{s}}{2} \frac{\partial^{2} \theta_{s}}{\partial x^{2}}=\omega \sin \theta_{s}-\Delta(x) \cos \theta_{s} .
$$

Here $D_{s}$ is the diffusion coefficient in the superconductor and $\Delta(x)$ is the superconducting pair potential. Notice that in the latter equation we have omitted the subscripts “ $\uparrow(\downarrow)$ )" because both equations are identical in the superconductor. 
Equations (4) and (5) should be complemented by the self-consistency equation for the superconducting order parameter $\Delta$,

$$
\Delta(x) \ln \frac{T_{c}}{T}=\pi T \sum_{\omega>0}\left(\frac{2 \Delta(x)}{\omega}-\sin \theta_{s \uparrow}-\sin \theta_{s \downarrow}\right),
$$

and by the boundary conditions at the outer boundary of the ferromagnet,

$$
\left(\frac{\partial \theta_{f}}{\partial x}\right)_{-d_{f} / 2}=0
$$

and at the $\mathrm{F} / \mathrm{S}$ interface, ${ }^{32}$

$$
\begin{gathered}
\xi_{n} \gamma\left(\frac{\partial \theta_{f}}{\partial x}\right)_{d_{f} / 2}=\xi_{s}\left(\frac{\partial \theta_{s}}{\partial x}\right)_{d_{f} / 2}, \\
\xi_{n} \gamma_{B 2}\left(\frac{\partial \theta_{f}}{\partial x}\right)_{d_{f} / 2}=\sin \left(\theta_{s}-\theta_{f}\right)_{d_{f} / 2},
\end{gathered}
$$

where $\gamma=\xi_{s} \sigma_{n} / \xi_{n} \sigma_{s}, \sigma_{s}$ is the conductivity of the $\mathrm{S}$ layer, and $\xi_{s}=\sqrt{D_{s} / 2 \pi T_{c}}$. The parameter $\gamma$ determines the strength of suppression of superconductivity in the right $\mathrm{S}$ lead near the interface compared to the bulk: No suppression occurs for $\gamma=0$, while strong suppression takes place for $\gamma \gg 1$. In our numerical calculations we will assume small $\gamma \ll 1$. Notice that the interface parameters do not depend on the spin direction. In other words we are not considering spin-active interfaces. In the case of spin-active barriers, one should use the boundary conditions introduced in Refs. 42-44, rather than the standard Kupriyanov-Lukichev boundary conditions Eqs. (8).

To complete the boundary problem we also set a boundary condition at $x=\infty$,

$$
\theta_{s}(\infty)=\arctan \frac{\Delta}{\omega}
$$

where the Green's functions acquire the well-known bulk BCS form. Equations (4)-(9) represent a closed set of equations that should be solved self-consistently. As will be discussed in the next section, the knowledge of the Green's function will allow us to compute the DOS at the outer F boundary.

\section{DENSITY OF STATES IN THE F/S BILAYER}

The DOS $N_{f}(E)$ normalized to the DOS in the normal state can be written as

$$
N_{f}(E)=\left[N_{f \uparrow}(E)+N_{f \downarrow}(E)\right] / 2,
$$

where $N_{f \uparrow(\downarrow)}(E)$ are the spin-resolved DOS written in terms of spectral angle $\theta$,

$$
N_{f \uparrow(\downarrow)}(E)=\operatorname{Re}\left[\cos \theta_{f \uparrow(\downarrow)}(i \omega \rightarrow E+i 0)\right] .
$$

To obtain $N_{f}$, we use a self-consistent two-step iterative procedure. ${ }^{37,45-47}$ In the first step we calculate the pair potential coordinate dependence $\Delta(x)$ using the self-consistency equation in the S layer, Eq. (6). Then, by proceeding to the analytical continuation in Eqs. (4) and (5) over the quasiparticle energy $i \omega \rightarrow E+i 0$ and using the $\Delta(x)$ dependence obtained in the previous step, we find the Green's functions by repeating the iterations until convergency is reached.

Before showing the numerical results we consider an analytic limiting case. If the F layer is thick enough $\left(d_{f} \gg \xi_{f 1}\right)$ and $\gamma=0$ in Eq. (8a), the DOS at the free boundary of the ferromagnet can be written as ${ }^{29,48}$

$$
N_{f \uparrow(\downarrow)}(E)=\operatorname{Re}\left[\cos \theta_{b \uparrow(\downarrow)}\right] \approx 1-\frac{1}{2} \operatorname{Re} \theta_{b \uparrow(\downarrow)}^{2} .
$$

Here $\theta_{b \uparrow(\downarrow)}$ is the value of $\theta_{f}$ at $x=-d_{f} / 2$, given by

$$
\theta_{b \uparrow(\downarrow)}=\frac{8 F(E)}{\sqrt{\left(1-\eta^{2}\right) F^{2}(E)+1}+1} \exp \left(-p \frac{d_{f}}{\xi_{f}}\right),
$$

where $\xi_{f}=\sqrt{D_{f} / h}$. In Eq. (13) we use the following notations,

$$
\begin{aligned}
p_{\uparrow(\downarrow)}= & \sqrt{2 / h} \sqrt{-i E_{R} \pm i h+1 / \tau_{m}}, \\
\eta_{\uparrow(\downarrow)}^{2}= & \left(1 / \tau_{m}\right)\left(-i E_{R} \pm i h+1 / \tau_{m}\right)^{-1}, \\
F(E)= & \frac{\Delta}{-i E_{R}+\sqrt{\Delta^{2}-E_{R}^{2}}}, \quad E_{R}=E+i 0 .
\end{aligned}
$$

Here, we again adopt the convention that a positive (negative) sign in front of $h$ corresponds to the spin-up state $\uparrow$ (spin-down state $\downarrow$ ). Hereafter we will write spin labels $\uparrow(\downarrow)$ explicitly only when needed.

From Eqs. (12) and (13) we obtain for the full DOS the following expression in the limit $d_{f} \gg \xi_{f 1}$,

$$
N_{f} \approx 1-\operatorname{Re} \sum_{\uparrow, \downarrow} \frac{16 F^{2}(E) \exp \left(-p \frac{2 d_{f}}{\xi_{f}}\right)}{\left(\sqrt{\left(1-\eta^{2}\right) F^{2}(E)+1}+1\right)^{2}} .
$$

At this point, we define the characteristic decay and oscillation lengths $\xi_{f 1,2 \uparrow(\downarrow)}$ as

$$
\begin{gathered}
p_{\uparrow(\downarrow)} / \xi_{f}=1 / \xi_{f 1 \uparrow(\downarrow)}+i \operatorname{sgn}(h \mp E) / \xi_{f 2 \uparrow(\downarrow)}, \\
\frac{1}{\xi_{f 1 \uparrow(\downarrow)}}=\frac{1}{\xi_{f}} \sqrt{\sqrt{\left(\frac{E \mp h}{h}\right)^{2}+\frac{1}{h^{2} \tau_{m}^{2}}+\frac{1}{h \tau_{m}}}} \\
\frac{1}{\xi_{f 2 \uparrow(\downarrow)}}=\frac{1}{\xi_{f}} \sqrt{\sqrt{\left(\frac{E \mp h}{h}\right)^{2}+\frac{1}{h^{2} \tau_{m}^{2}}}-\frac{1}{h \tau_{m}}} .
\end{gathered}
$$

In the absence of magnetic scattering both lengths coincide and are equal to $\xi_{f} \sqrt{h /|E \mp h|}$ for different spin orientations. One can rewrite Eq. (15) in the following form,

$$
\begin{aligned}
N_{f} \approx & 1-\sum_{\uparrow, \downarrow} \exp \left(-\frac{2 d_{f}}{\xi_{f 1}}\right)\left[\mathcal{A} \sin \left(\chi+\frac{2 d_{f}}{\xi_{f 2}}\right)\right. \\
& \left.+\mathcal{B} \cos \left(\chi+\frac{2 d_{f}}{\xi_{f 2}}\right)\right],
\end{aligned}
$$

where the coefficients $\mathcal{A}, \mathcal{B}$, and $\chi$ can be obtained by expansion of the real part in Eq. (15); only two of them are independent. This form explicitly shows the damped oscillatory behavior of superconducting correlations in the F layer. The lengths $\xi_{f 1,2}$ are also the lengths of decay and oscillations of the critical current in SIFS junctions [see Eqs. (26) in Ref. 29]. The period of the DOS oscillations is approximately twice smaller than the period of the critical current oscillations and the exponential decay is approximately twice faster than the decay of the critical current. ${ }^{29}$

Now we turn to the exact numerical solution. The obtained energy dependencies of the DOS at the free F boundary of the 


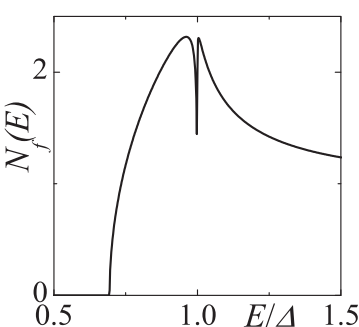

(a)

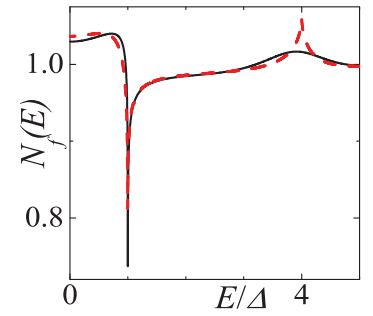

(c)

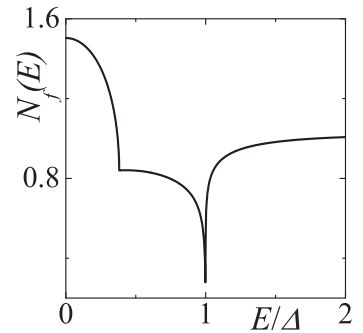

(b)

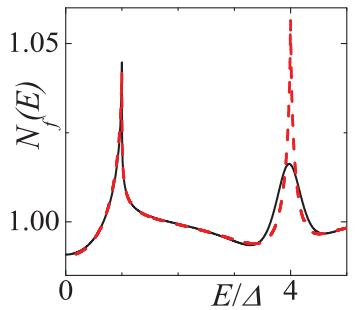

(d)
FIG. 2. (Color online) DOS $N_{f}(E)$ on the free boundary of the $\mathrm{F}$ layer in the $\mathrm{F} / \mathrm{S}$ bilayer calculated numerically in the absence of magnetic scattering $\left(1 / \tau_{m} \Delta=0\right)$ for different values of the $\mathrm{F}$ layer thickness $d_{f}, h / \Delta=4, T=0.1 T_{c}$. Parameters of the $\mathrm{F} / \mathrm{S}$ interface are $\gamma=\gamma_{B 2}=0.01$. (a) $d_{f} / \xi_{n}=0.5$. (b) $d_{f} / \xi_{n}=1$. (c) $d_{f} / \xi_{n}=$ 2. (d) $d_{f} / \xi_{n}=3$. The approximate analytical solution, Eq. (15), is shown by dashed red lines.

F/S bilayer are presented in Figs. 2, 3, and 4. The exchange field is chosen such that $h>\Delta$, which corresponds to the experimental situation.

Figure 2 shows the DOS energy dependence for different $d_{f}$ in the absence of magnetic scattering. At small $d_{f}$ we observe the DOS double peak due to the Zeeman splitting of the BCS peak at $E=\Delta$. Most probably in the experiments, the BCS Zeeman-split peak as presented in Fig. 2(a) will be seen as a single peak due to many-body interaction effects, which introduce a finite lifetime (damping) of the quasiparticles. We also observe that at small $d_{f}$ and relatively small exchange field $h$, full DOS turns to zero inside a minigap, which vanishes with the increase of $d_{f}$.

The minigap also exists in the normal metal $(\mathrm{N})$ DOS in the $\mathrm{S} / \mathrm{N}$ bilayers. If the thickness $d_{n}$ of the normal metal is larger than the coherence length, the characteristic scale of the minigap is set by the Thouless energy, $E_{T h}=D_{n} / d_{n}^{2}$, where $D_{n}$ and is the diffusion coefficient of the normal metal. ${ }^{46}$ In the F layer of the S/F bilayer, the exchange field $h$ shifts the DOS for the two spin subbands in opposite directions; therefore the critical value $h_{c}$ of the exchange field at which the minigap in the spectrum closes can be roughly estimated as ${ }^{49}$

$$
h_{c} \sim E_{T h}, \quad E_{T h}=D_{f} / d_{f}^{2} .
$$

This equation shows the qualitative tendency that for smaller $d_{f}$ a higher $h$ is needed to close the minigap [see also Fig. 3(a)]. The estimation, Eq. (18), is only valid in the absence of magnetic scattering, since $\tau_{m}$ also influences the miniga ${ }^{50}$ [see also Fig. 4(a)].

In Fig. 2 we also observe that after the minigap closes, the DOS at the Fermi energy $N_{f}(0)$ rapidly increases to values larger than unity with further increase of $d_{f}$; then it

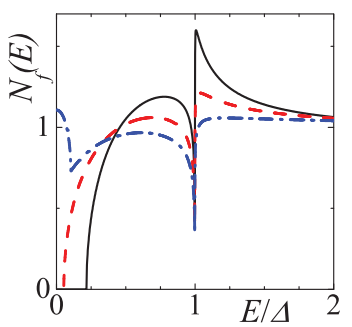

(a)

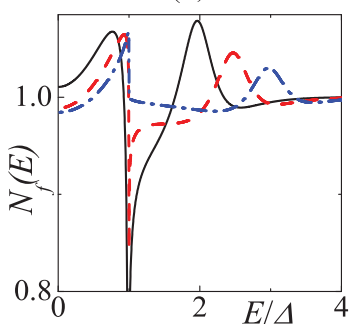

(c)

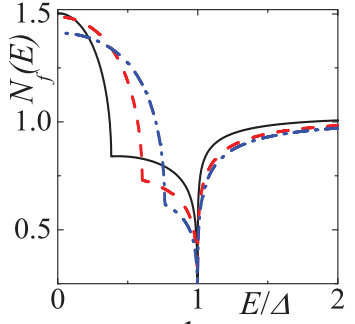

(b)

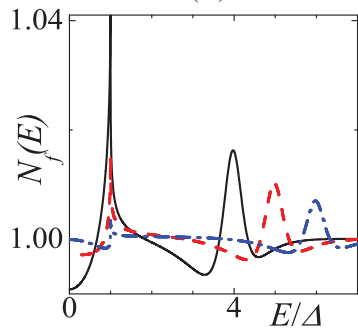

(d)
FIG. 3. (Color online) DOS $N_{f}(E)$ on the free boundary of the $\mathrm{F}$ layer in the $\mathrm{F} / \mathrm{S}$ bilayer calculated numerically in the absence of magnetic scattering $\left(1 / \tau_{m} \Delta=0\right)$ for different values of the exchange field $h$. Parameters of the F/S interface are $\gamma=\gamma_{B 2}=0.01, T=$ $0.1 T_{c}$. Plots (a) and (b): $d_{f} / \xi_{n}=1$; plots (c) and (d): $d_{f} / \xi_{n}=3$. For plots (a) and (c) solid black line corresponds to $h / \Delta=2$, dashed red line to $h / \Delta=2.5$, dash-dotted blue line to $h / \Delta=3$. For plots (b) and (d) solid black line corresponds to $h / \Delta=4$, dashed red line to $h / \Delta=5$, dash-dotted blue line to $h / \Delta=6$.

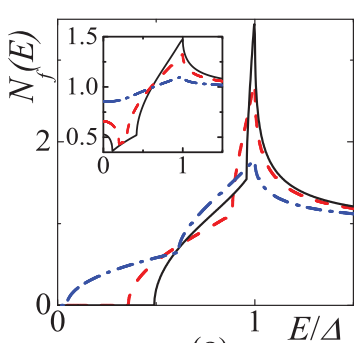

(a)

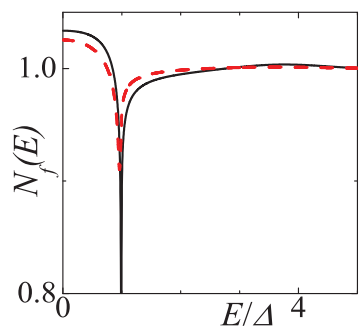

(c)

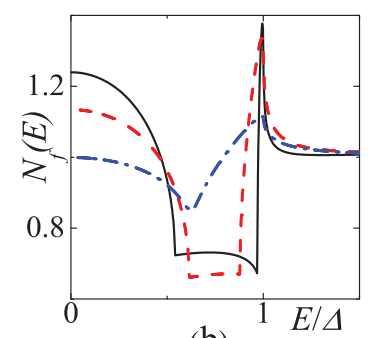

(b)

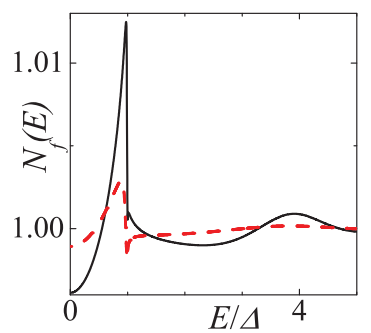

(d)
FIG. 4. (Color online) DOS $N_{f}(E)$ at the free boundary of the $\mathrm{F}$ layer in the $\mathrm{F} / \mathrm{S}$ bilayer calculated numerically for $\alpha_{m}=1 / \tau_{m} \Delta=$ 0.5 (solid black line), $\alpha_{m}=1$ (dashed red line), and $\alpha_{m}=3$ (dashdotted blue line) for different values of the F layer thickness $d_{f}, h=$ $4 \Delta, T=0.1 T_{c}$. Parameters of the F/S interface are $\gamma=\gamma_{B 2}=0.01$. (a) $d_{f} / \xi_{n}=0.5$. (b) $d_{f} / \xi_{n}=1$. (c) $d_{f} / \xi_{n}=2$. (d) $d_{f} / \xi_{n}=3$. For plots (c) and (d) the curves with $\alpha_{m}=3$ are not shown since they are of the order of unity at corresponding scale. Inset of the plot (a): $N_{f}(E)$ dependence for $d_{f} / \xi_{n}=0.5$ for higher values of $\alpha_{m} ; \alpha_{m}=5$ (solid black line), $\alpha_{m}=7$ (dashed red line), $\alpha_{m}=15$ (dash-dotted blue line). 


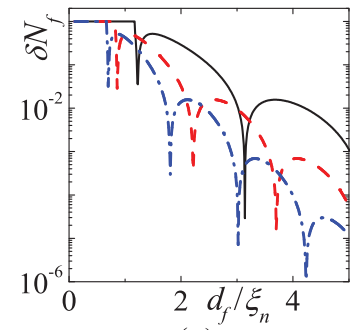

(a)

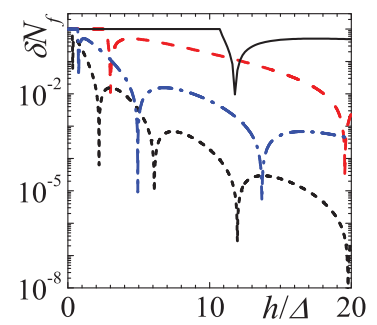

(c)

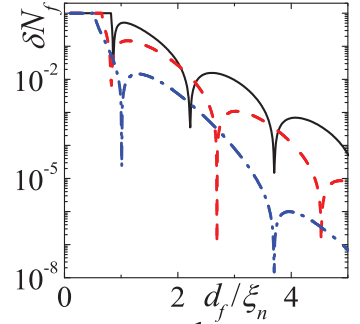

(b)

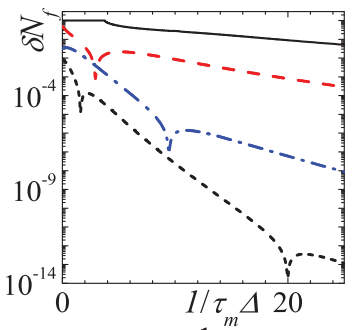

(d)
FIG. 5. (Color online) Dependence of $\delta N_{f}$ as a function of the $\mathrm{F}$ layer thickness $d_{f}$ for different exchange fields (a) and magnetic scattering times (b); dependence of $\delta N_{f}$ as a function of exchange field (c) and magnetic scattering time (d) for different $d_{f}$. The temperature $T=0.1 T_{C}$. Parameters of the $\mathrm{F} / \mathrm{S}$ interface are $\gamma=\gamma_{B 2}=0.01$. (a) No magnetic scattering $\left(1 / \tau_{m} \Delta=0\right), h / \Delta=$ 2 (black solid line), $h / \Delta=4$ (red dashed line), $h / \Delta=6$ (blue dash-dotted line). (b) $h / \Delta=4, \alpha_{m}=1 / \tau_{m} \Delta=0$ (black solid line), $\alpha_{m}=1$ (red dashed line), $\alpha_{m}=3$ (blue dash-dotted line). (c) No magnetic scattering, $d_{f} / \xi_{n}=0.5$ (black solid line), $d_{f} / \xi_{n}=1$ (red dashed line), $d_{f} / \xi_{n}=2$ (blue dash-dotted line), $d_{f} / \xi_{n}=3$ (black short-dashed line). (d) $h / \Delta=4, d_{f} / \xi_{n}=0.5$ (black solid line), $d_{f} / \xi_{n}=1$ (red dashed line), $d_{f} / \xi_{n}=2$ (blue dash-dotted line), $d_{f} / \xi_{n}=3$ (black short-dashed line).

oscillates around unity while its absolute value exponentially approaches unity (see also Fig. 5). This is the well-known damped oscillatory behavior of the DOS in F/S bilayers. Experimental evidence for such behavior was provided by Kontos et al. ${ }^{51}$ In the case of a long enough ferromagnetic layer we also observe the DOS peak at $E=h$, which was previously discussed in Ref. 52. A similar effect was also discussed in $\mathrm{N} / \mathrm{F} / \mathrm{S}$ structures, where it was shown that a zero energy peak appears in DOS if $E_{T h}=h .^{53}$

We also show in Fig. 2 the analytical approximation, Eq. (15), which is in good agreement with the numerical result for thick enough ferromagnetic layers. In the numerically obtained curves the peak at $E=h$ is smeared because of finite $\gamma=0.01$ for the transparent $\mathrm{F} / \mathrm{S}$ interface at $x=d_{f} / 2$.

In the absence of magnetic scattering we rewrite the analytical DOS expression, Eq. (15), for $E \geqslant \Delta$ in the following way,

$$
\begin{aligned}
N_{f}(E)= & +\sum_{ \pm} \frac{16 \Delta^{2} \cos \left(\frac{2 d_{f}}{\xi_{f}} \sqrt{\frac{|E \pm h|}{h}}\right)}{(E+\varepsilon)(\sqrt{E+\varepsilon}+\sqrt{2 \varepsilon})^{2}} \\
& \times \exp \left(-\frac{2 d_{f}}{\xi_{f}} \sqrt{\frac{|E \pm h|}{h}}\right),
\end{aligned}
$$

where $\varepsilon=\sqrt{E^{2}-\Delta^{2}}$. We can clearly see the exponential asymptotic of the peak at $E=h$ from the Eq. (19). We should keep in mind that Eq. (19) is valid for large $d_{f} / \xi_{f}$, but nevertheless we may qualitatively understand why we do not see the peak at $E=h$ for a small ratio of $d_{f} / \xi_{f}$ : If this factor is small the variation of the exponent $\exp \left\{-2\left(d_{f} / \xi_{f}\right) \sqrt{|E-h| / h}\right\}$ near the point $E=h$ is also small. The peak is observable only for $h$ of the order of a few $\Delta$. For larger exchange fields the peak is very difficult to observe, since the energy dependent prefactor of the exponent in Eq. (19) decays as $E^{-2}$ for $E \gg \Delta$.

Figure 3 shows the DOS energy dependence for different values of the exchange field $h$ in the absence of magnetic scattering. For stronger exchange field the minigap closes at smaller $d_{f}$, in qualitative correspondence with Eq. (18). From numerical calculations we obtain the following condition [see also Fig. 5(c)], valid for $\tau_{m}^{-1} \sim 0,{ }^{54}$

$$
h_{c} \approx 0.77 E_{T h} \approx 2.71 \Delta\left(\frac{\xi_{n}}{d_{f}}\right)^{2} .
$$

In Fig. 3 we also observe the peak at $E=h$; at large enough exchange fields its amplitude can be much larger than the amplitude of the peak at $E=\Delta$ [see Fig. 3(d), blue dash-dotted curve]. The existence of the DOS peak at $E=h$ gives a possibility to measure the exchange field directly in experiment by measuring the $\mathrm{F} / \mathrm{S}$ bilayer DOS in compounds with small magnetic scattering (since magnetic scattering is smearing the peak, see below). For example, in Ref. 55 were reported exchange fields for $\mathrm{Pd}_{1-x} \mathrm{Ni}_{x}$ with different $\mathrm{Ni}$ concentration, obtained by a fitting procedure. Considering $\mathrm{Nb}$ as a superconductor with $\Delta=1.3 \mathrm{meV}$, we can estimate the exchange field in $\mathrm{Pd}_{1-x} \mathrm{Ni}_{x}$ : For $7 \%$ of $\mathrm{Ni}$ fitting gives $h=$ $2.8 \mathrm{meV}$, which is $2.2 \Delta$, and for $11.5 \%$ of $\mathrm{Ni} h=3.9 \mathrm{meV}$, which is $3 \Delta .{ }^{55} \mathrm{It}$ is interesting to use direct measurements of the DOS peak at $E=h$ to check these fitting predictions of Ref. 55.

Ferromagnetic metals with exchange fields of the order of few $\Delta$ are crucially important for the fabrication of SIFS junctions, valid for superconducting logic applications. Presently used ferromagnets have $h \gg \Delta$, and therefore short oscillation length [see Eq. (16c)], which makes it difficult to control the F layer thickness. In already existing SIFS structures the roughness is often larger than desired precision of $d_{f} .{ }^{56} \mathrm{We}$ hope that our results will trigger the experimental activity in finding ferromagnetic alloys with $h$ of the order of few $\Delta$.

Figure 4 shows the DOS energy dependence for different values of magnetic scattering time. Similarly to Fig. 3, for stronger magnetic scattering the minigap closes at smaller $d_{f}$. Also the DOS peak at $E=h$, visible for a long enough ferromagnetic layer, is smeared. The analytical solution (not shown), Eq. (15), also agrees quite well with the numerical results for $d_{f} \gg \xi_{f 1}$.

Although our results are obtained for weak ferromagnets, they can in certain cases be extended for ferromagnets with strong exchange fields, $h \gg \Delta$. In the absence of magnetic scattering the Usadel equation in energy representation, Eq. (4), can be rewritten as

$$
\frac{i}{2} \frac{\partial^{2} \theta_{f \uparrow(\downarrow)}}{\partial y^{2}}=\left(\frac{E}{h} \mp 1\right) \sin \theta_{f \uparrow(\downarrow)},
$$

where $y=x / \xi_{f}$ is the dimensionless coordinate. In the case of $h \gg \Delta$, we can neglect the first term on the right-hand side 
of Eq. (21) to obtain the subgap DOS. Thus, in that case the subgap structure scales with the length $\xi_{f}$ and for example the results presented in Fig. 2 for $h=4 \Delta$ also describe the DOS in the case of a high exchange field if one scales $d_{f}$ correspondingly. This procedure does not apply however for $h=2 \Delta$ (see Fig. 3), since in that case one cannot simply neglect the term $E / h$ in Eq. (21).

To show explicitly the aforementioned DOS oscillations at the Fermi energy ${ }^{51}$ in Fig. 5, where we plot the numerically calculated function

$$
\delta N_{f}\left(d_{f}, h, \tau_{m}\right)=\left|1-N_{f 0}\right|, \quad N_{f 0}=N_{f}(E=0) .
$$

Using Eqs. (12)-(17) and (22) we get the analytical expression for the function $\delta N$, valid for $d_{f} \gg \xi_{f 1}$,

$$
\delta N=32\left|\operatorname{Re}\left[\frac{1}{\left(\sqrt{2-\eta_{0}^{2}}+1\right)^{2}} \exp \left(-p_{0} \frac{2 d_{f}}{\xi_{f}}\right)\right]\right|,
$$

where

$$
\begin{gathered}
p_{0}=\sqrt{2 / h} \sqrt{i h+1 / \tau_{m}}, \\
\eta_{0}=\left(1 / \tau_{m}\right)\left(i h+1 / \tau_{m}\right)^{-1} .
\end{gathered}
$$

At vanishing magnetic scattering we obtain

$$
\delta N=\frac{32}{3+2 \sqrt{2}}\left|\cos \left(\frac{2 d_{f}}{\xi_{f}}\right) \exp \left(-\frac{2 d_{f}}{\xi_{f}}\right)\right|,
$$

in which case the characteristic lengths of decay and oscillations are equal to $\xi_{f}$.

The dependence of $\delta N_{f}\left(d_{f}\right)$ on the ferromagnetic layer thickness $d_{f}$ at different values of exchange field and magnetic scattering time is presented in Figs. 5(a) and 5(b). From Fig. 5(a) we can see that with increasing exchange field $h$ the minigap closes at smaller $d_{f}$ in agreement with Eq. (20), the period of the DOS oscillations at the Fermi energy decreases, and the damped exponential decay occurs faster. This is easy to see from Eq. (25), since in the absence of magnetic scattering $\delta N$ depends on $h$ only as a function of $\xi_{f}$.

From Fig. 5(b) we can see that with increasing $\alpha_{m}=1 / \tau_{m} \Delta$ the period of the DOS oscillations on the contrary increases, although the minigap also closes at smaller $d_{f}$ and the damped exponential decay occurs faster. To understand this behavior we rewrite here the decay and oscillation lengths, Eq. (16), at the Fermi energy,

$$
\begin{gathered}
\frac{1}{\xi_{f 1}}=\frac{1}{\sqrt{D_{f}}} \sqrt{\sqrt{h^{2}+\frac{1}{\tau_{m}^{2}}}+\frac{1}{\tau_{m}}} \\
\frac{1}{\xi_{f 2}}=\frac{1}{\sqrt{D_{f}}} \sqrt{\sqrt{h^{2}+\frac{1}{\tau_{m}^{2}}}-\frac{1}{\tau_{m}}} .
\end{gathered}
$$

We see from these equations that with increasing $\alpha_{m}$ the length of decay $\xi_{f 1}$ decreases, while the length of oscillations $\xi_{f 2}$ increases.

The dependencies of $\delta N_{f}$ on exchange field and magnetic scattering time are presented in Figs. 5(c) and 5(d), correspondingly. In Fig. 5(c) we see oscillations of the DOS at the Fermi energy $\delta N_{f}(h)$ around unity with increasing exchange field in the absence of magnetic scattering. In Fig. 5(d) we show the function $\delta N\left(\tau_{m}\right)$. It is interesting to note that its behavior can be both oscillatory and also monotonous. When the parameter $\alpha_{m}$ increases starting from the minigap state (black solid curve) it is totally monotonous: Increasing $\alpha_{m}$ the minigap closes and the DOS starts to increase to unity, but never overshoots unity [we checked this up to $\alpha_{m}=80$, see also Fig. 4(a)]. If we start from the state where the minigap is already closed, we first observe oscillations, but then again a switch to monotonous behavior. For intermediate F-layer thicknesses we see just one oscillation and then DOS monotonously approaches unity (we checked this up to $\alpha_{m}=80$ ), while for thicker ferromagnets $\left(d_{f} / \xi_{n}=3\right)$ we observe two oscillations and then a monotonic behavior.

The dependencies $\delta N(h)$ and $\delta N\left(\tau_{m}\right)$ can be important if in the experiment the material properties of the ferromagnetic interlayer, i.e., exchange field $h$ and magnetic scattering time $\tau_{m}$, can vary with some external parameter, for example temperature, magnetic field, etc.

Before turning to the calculation of the CVC we discuss briefly a recent experiment ${ }^{57}$ in which a pronounced double peak in the DOS of $\mathrm{Ni} / \mathrm{Nb}$ bilayers was reported. This double peak cannot be explained within our model based on the Zeeman splitting. The reason for the double peak in Ref. 57 remains controversial. In Ref. 44 it was numerically fitted by adding an extra parameter to the model, characterizing spin-active interfaces. However, this fit is far from being satisfactory. Nevertheless there is another feature of the DOS observed in Ref. 57 which can be explained within our model: By increasing $d_{f}$ the "normal" peak at $E=\Delta$ [which is the BCS Zeeman-split peak in Fig. 2(a)] is "inverted" [Figs. 2(b) and 2(c)] and becomes "normal" again [Fig. 2(d)] as $d_{f}$ is further increased. According to our model, at $E=\Delta$ Eq. (19) reduces to the following expression,

$$
\begin{aligned}
N_{f}(\Delta)= & 1+16 \sum_{ \pm} \cos \left(\frac{2 d_{f}}{\xi_{f}} \sqrt{\frac{h \pm \Delta}{h}}\right) \\
& \times \exp \left(-\frac{2 d_{f}}{\xi_{f}} \sqrt{\frac{h \pm \Delta}{h}}\right) .
\end{aligned}
$$

This expression explains the inversion of the peak at $E=\Delta$ as a function of $d_{f}$. The peak is "normal" ("inverted") if the DOS at $\Delta$ is larger (smaller) than unity. This variation is due to the sign of the cosine function in Eq. (27), which depends on the $d_{f} / \xi_{f}$ ratio.

\section{CURRENT-VOLTAGE CHARACTERISTICS OF A SIFS JUNCTION}

In this section we calculate the current-voltage characteristics (CVC) of a SIFS junction at low temperature, $T=0.1 T_{c}$, using Eq. (1) and DOS $N_{f}\left(E, d_{f}, h, \tau_{m}\right)$ numerically obtained in the previous section.

Figure 6 shows the CVC of a SIFS junction in the absence of magnetic scattering. For comparison we also present the CVC of a SINS tunnel junction, i.e., a junction with a normal metal interlayer instead of a ferromagnet $(h=0)$. SINS structures were studied previously in Ref. 46. We observe several features of SIFS CVC which are the signatures of the proximity effect in the $\mathrm{S} / \mathrm{F}$ bilayer. 


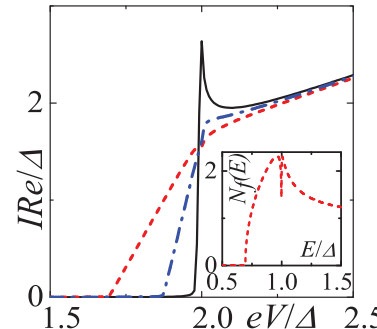

(a)

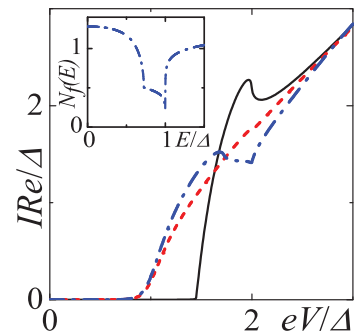

(c)

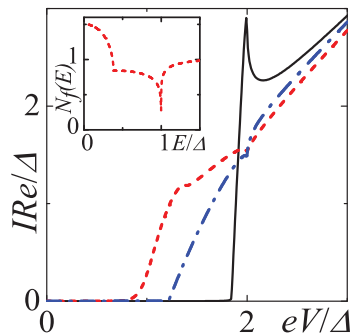

(b)

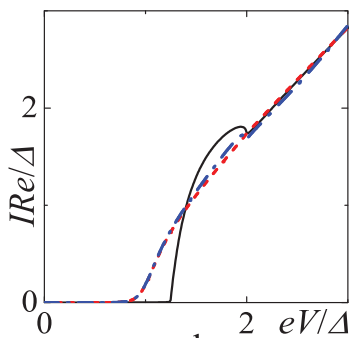

(d)
FIG. 6. (Color online) Current-voltage characteristics of a SIFS junction in the absence of magnetic scattering for different values of the F-layer thickness $d_{f}$. The temperature $T=0.1 T_{c}$. The exchange field $h=0$ (black line, which corresponds to the case of a SINS junction), $h / \Delta=2$ (blue dash-dotted line), and $h / \Delta=4$ (red short-dashed line). (a) $d_{f} / \xi_{n}=0.5$. (b) $d_{f} / \xi_{n}=1$. (c) $d_{f} / \xi_{n}=2$. (d) $d_{f} / \xi_{n}=3$. Insets in (a), (b), and (c) are explained in the text.

For a thin enough F layer we observe the "kink" on the $\mathrm{CVC}$ at $e V \approx 2 \Delta$ [Fig. 6(a)], which corresponds to the case when the DOS $N_{f}(E)$ exhibits a pronounced minigap. The corresponding DOS energy dependence $\left(h / \Delta=4, d_{f} / \xi_{n}=\right.$ $0.5)$ is shown in the inset. We can also see that for a certain range of parameters the CVC of a SIFS junction exhibit a nonmonotonic "wave" behavior. We can observe it for $h / \Delta=4$ (red short-dashed line) in Fig. 6(b) and for $h / \Delta=2$ (blue dash-dotted line) in Fig. 6(c). This behavior corresponds to the case when the DOS $N_{f}(E)$ minigap is already closed and the $N_{f}(0)$ at the Fermi energy is larger than unity. The corresponding DOS energy dependencies are presented in the insets of the plots in Figs. 6(b) and 6(c).

At large enough $d_{f}$ and exchange fields the DOS $N_{f}(E) \approx$ 1 and the current, Eq. (1), is given by the same equation as the current in the NIS tunnel junction,

$$
I=\frac{1}{e R} \int_{-\infty}^{\infty} d E N_{s}(E)[f(E-e V)-f(E)]
$$

At small temperature $T \ll T_{c}$, this equation is well approximated by taking $T=0$,

$$
\begin{aligned}
I & =\Theta(e V-\Delta) \frac{1}{e R} \int_{\Delta}^{e V} \frac{E d E}{\sqrt{E^{2}-\Delta^{2}}} \\
& =\Theta(e V-\Delta) \frac{\sqrt{(e V)^{2}-\Delta^{2}}}{e R} .
\end{aligned}
$$

The red short-dashed line in Fig. 6(d) $\left(d_{f} / \xi_{n}=3, h / \Delta=\right.$ 4 , no magnetic scattering) almost coincides with this result

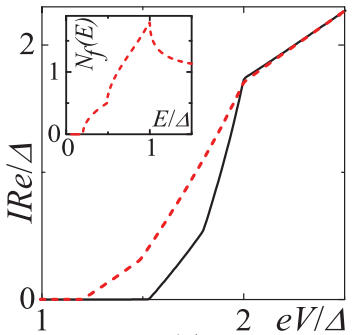

(a)

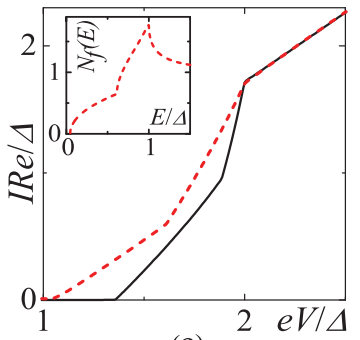

(c)

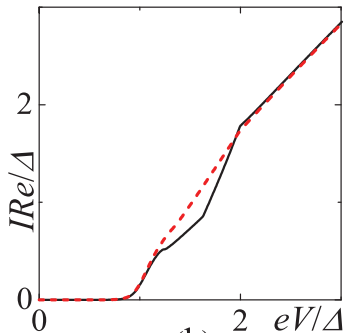

(b)

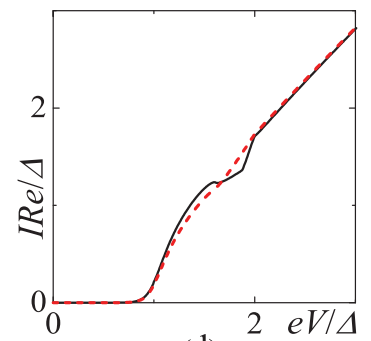

(d)
FIG. 7. (Color online) Current-voltage characteristics of a SIFS junction for $1 / \tau_{m} \Delta=1$ (black solid lines) and $1 / \tau_{m} \Delta=3$ (red shortdashed lines) for different values of the F-layer thickness $d_{f}$. The temperature $T=0.1 T_{c}$. The exchange field $h / \Delta=2$ [plots (a) and (b)] and $h / \Delta=4$ [plots (c) and (d)]. The thickness $d_{f} / \xi_{n}=0.5$ [plots (a) and (c)] and $d_{f} / \xi_{n}=1$ [plots (b) and (d)]. Insets in (a) and (c) are explained in the text.

except for the small region $e V \approx \Delta$, since for our numerically calculated curves we fix temperature $T=0.1 T_{c} \ll T_{c}$.

Figure 7 shows current-voltage characteristics of a SIFS junction in case of finite magnetic scattering. Here for thin F layers we observe a "double-kink" structure, see Figs. 7(a) and 7(c). It corresponds to the DOS $N_{f}(E)$ with small minigap and finite subgap value smaller than unity. Such a DOS structure is typical in the presence of magnetic scattering and thin enough ferromagnetic interlayer, see Fig. 4(a). The corresponding DOS energy dependencies are presented in the insets of the plots in Figs. 6(a) $\left[1 / \tau_{m} \Delta=3, h / \Delta=2, d_{f} / \xi_{n}=\right.$ $0.5]$ and 6(b) $\left[1 / \tau_{m} \Delta=3, h / \Delta=4, d_{f} / \xi_{n}=0.5\right]$. For finite magnetic scattering the nonmonotonic features of CVC are smeared. We do not show the curves for $d_{f} / \xi_{n} \geqslant 2$, since they do not significantly differ from the curves obtained from Eq. (29).

Figures 6(a) and 7(a) show that for a thin enough ferromagnetic layer the current has an onset in the interval $[\Delta, 2 \Delta]$ (for temperatures $T \ll T_{C}$ ). The value of this onset, according to Eq. (2), is $\Delta+E_{g}$, where $E_{g}$ is the DOS minigap, $0<E_{g}<$ $\Delta$. Increasing exchange field, magnetic scattering, and/or Flayer thickness, the minigap closes and the current turns to zero at $e V<\Delta$, having an onset at $e V=\Delta$. The dependence of the minigap $E_{g}$ on the parameters characterizing the material properties of the ferromagnetic interlayer is discussed in Sec. III.

We conclude that we observe interesting features in the SIFS CVC if the DOS $N_{f}(E)$ near the insulating barrier has a nontrivial shape in the subgap region. In the case when $N_{f} \approx 1$, these features disappear and the CVC coincide with those of the NIS tunnel junction, Eq. (28). 
(a)

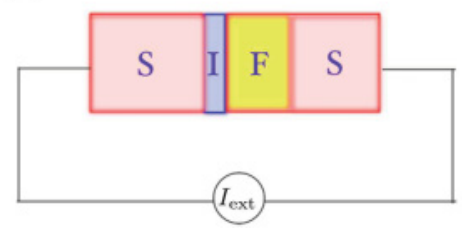

(b)

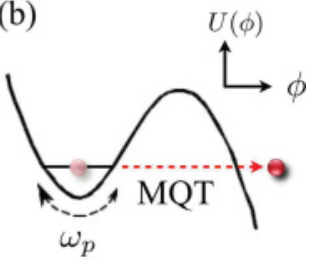

FIG. 8. (Color online) (a) Schematic of a current biased SIFS Josephson junction. $I_{\text {ext }}$ is the external bias current. (b) Potential $U(\phi)$ vs the phase difference $\phi$ between two superconductors. $\omega_{p}$ is the Josephson plasma frequency of the junction.

\section{MACROSCOPIC QUANTUM TUNNELING IN A SIFS JUNCTION}

In this section, motivated by experimental studies on the (thermal and quantum) switching ${ }^{58}$ and quantum coherent oscillations $^{26}$ in SFS and SIFS junctions, we calculate the MQT escape rate in a current-biased SIFS junction as shown in Fig. 8(a). The CVC obtained in the previous section enable us to investigate the influence of the quasiparticle dissipation on MQT.

It is important to note that MQT can be used as a measurement process of a superconducting phase qubit. ${ }^{59}$ Thus, the calculation of the MQT rate by taking into account the quasiparticle dissipation will be very important for analyzing the fidelity of the measurement process for phase qubits. In the following calculation, for simplicity, we have ignored the influence from an environmental circuit on MQT which can be experimentally reduced by a noise filtering technique. ${ }^{60}$

The partition function of a junction can be described by an imaginary-time functional integral over the macroscopic variable (the phase difference $\phi$ between two superconductors); ${ }^{61-64}$ i.e.,

$$
Z=\int D \phi(\tau) \exp \left(-\frac{S_{\mathrm{eff}}[\phi]}{\hbar}\right) .
$$

In the strong insulating barrier limit, i.e., $\gamma_{B 1} \gg 1$, the effective action $S_{\text {eff }}$ is given by

$$
\begin{aligned}
S_{\mathrm{eff}}[\phi] & =\int_{0}^{\hbar \beta} d \tau\left[\frac{M}{2}\left(\frac{\partial \phi(\tau)}{\partial \tau}\right)^{2}+U(\phi)\right]+S_{\alpha}[\phi], \\
S_{\alpha}[\phi] & =-\int_{0}^{\hbar \beta} d \tau \int_{0}^{\hbar \beta} d \tau^{\prime} \alpha\left(\tau-\tau^{\prime}\right) \cos \frac{\phi(\tau)-\phi\left(\tau^{\prime}\right)}{2} .
\end{aligned}
$$

In this equation, $\beta=1 / k_{B} T, M=C(\hbar / 2 e)^{2}$ is the mass $(C$ is the capacitance of the junction) and the potential $U(\phi)$ can be described by a tilted washboard potential [Fig. 8(b)]; i.e.,

$$
U(\phi)=-E_{J}\left[\operatorname{sgn}\left(I_{c}\right) \cos \phi+y \phi\right],
$$

with $y \equiv I_{\text {ext }} /\left|I_{c}\right|$, where $E_{J}=\hbar\left|I_{c}\right| / 2 e$ is the Josephson coupling energy, $I_{c}$ is the Josephson critical current, and $I_{\text {ext }}$ is the external bias current. The dissipation kernel $\alpha(\tau)$ is related to the quasiparticle current $I$ under constant bias voltage $V$ by

$$
\alpha(\tau)=\frac{\hbar}{e} \int_{0}^{\infty} \frac{d \omega}{2 \pi} \exp (-\omega \tau) I\left(V=\frac{\hbar \omega}{e}\right)
$$

at zero temperature. ${ }^{61-63}$

As clearly seen from Figs. 6 and 7, the CVC has a gap structure due to the isotropic superconducting gap in the left superconductor electrode. In such a case, the dissipation kernel $\alpha$ decays exponentially as a function of imaginary time $\tau$ for $|\tau| \gg \hbar / \Delta$. The typical dynamical time scale of the macroscopic variable $\phi$ is of the order of the inverse Josephson plasma frequency $\omega_{p}=\sqrt{2 e\left|I_{c}\right| / \hbar C}\left(1-y^{2}\right)^{1 / 4}$ which is much smaller than $\Delta$. Thus, the phase varies slowly with the time scale given by $\hbar / \Delta$, and we can expand $\phi(\tau)-\phi\left(\tau^{\prime}\right)$ in Eq. (31b) about $\tau=\tau^{\prime}$. This gives

$$
S_{\alpha}[\phi] \approx \frac{\delta C}{2} \int_{0}^{\hbar \beta} d \tau\left[\frac{\hbar}{2 e} \frac{\partial \phi(\tau)}{\partial \tau}\right]^{2},
$$

where

$$
\delta C=2\left(\frac{2 e}{\hbar}\right)^{2} \int_{0}^{\infty} d \tau \alpha(\tau) \tau^{2} .
$$

Hence, the dissipation action $S_{\alpha}$ acts as a kinetic term so that the effect of the quasiparticles results in an increase of the capacitance, $C \rightarrow C+\delta C \equiv C_{\text {ren }}$.

In the case of a thin ferromagnetic layer $\left(d_{f}=0.5 \xi_{n}\right)$ we numerically obtain $\delta C \approx \hbar / \Delta R$ for CVC presented in Figs. 6(a), 7(a), and 7(c) ["kink" and "double-kink" structures]. For a thick ferromagnetic layer we can use Eq. (29) to calculate $\delta C$,

$$
\delta C=\frac{4 \hbar}{\pi \Delta R} \int_{0}^{\infty} d x x^{2} \int_{1}^{\infty} d z e^{-x z} \sqrt{z^{2}-1} \approx \frac{2 \hbar}{\Delta R} .
$$

For intermediate $d_{f}$ we numerically find $\delta C \approx(1-2) \hbar / \Delta R$. Considering $\mathrm{Nb}$ as a superconductor $(\Delta=1.3 \mathrm{meV})$ we therefore obtain $\delta C \approx(0.5-1) r^{-1} \mathrm{pF}$, where $r$ is the junction resistance $R$ in $\Omega$. To insure a small dissipative correction of capacitance, $\delta C \ll C$, we have a constraint,

$$
R C \gg \hbar / \Delta
$$

i.e., the typical time constant $R C$ of a SIFS junction should be much larger than the dynamical damping scale for the dissipation kernel $\alpha(\tau)$. For example, in Ref. 13 the following parameters of a Nb/ $\mathrm{Al}_{2} \mathrm{O}_{3} / \mathrm{Ni}_{0.6} \mathrm{Cu}_{0.4} / \mathrm{Nb}$ SIFS junction were reported, $C=800 \mathrm{pF}$ and $R=55 \mathrm{~m} \Omega$, which correspond to $\delta C \approx 10-18 \mathrm{pF}$. Thus, even for the low resistive tunnel barrier in Ref. 13, we have the condition Eq. (37) satisfied.

In order to see the effect of the quasiparticle dissipation on macroscopic quantum dynamics, we will investigate MQT in current-biased SIFS junctions. The MQT escape rate $\Gamma$ from the metastable potential at zero temperature is given by ${ }^{66}$

$$
\Gamma=\lim _{\beta \rightarrow \infty} \frac{2}{\beta} \operatorname{Im} \ln Z .
$$

By using the Caldeira and Leggett theory, ${ }^{68}$ the MQT rate is approximated as

$$
\Gamma=\frac{\hat{\omega}_{p}}{2 \pi} \sqrt{120 \pi B} \exp (-B),
$$

where

$$
\hat{\omega}_{p}=\sqrt{\frac{2 e\left|I_{c}\right|}{\hbar C_{\text {ren }}}}\left(1-y^{2}\right)^{1 / 4}
$$


is the renormalized Josephson plasma frequency and $B=$ $S_{\text {eff }}\left[\phi_{B}\right] / \hbar$ is the bounce exponent, which is the value of the action $S_{\text {eff }}$ evaluated along the bounce trajectory $\phi_{B}(\tau)$. The analytic expression for the bounce exponent is given by

$$
B=\frac{12}{5 e} \sqrt{\frac{\hbar}{2 e}\left|I_{c}\right| C_{\text {ren }}}\left(1-y^{2}\right)^{5 / 4} .
$$

At high temperatures, the thermally activated decay dominates the escape process. Then the escape rate is given by the Kramers formula, $\Gamma=\left(\hat{\omega}_{p} / 2 \pi\right) \exp \left(-U_{0} / k_{B} T\right)$, where $U_{0}$ is the barrier height. ${ }^{66}$ Below the crossover temperature $T^{*}$, the escape process is dominated by MQT. In the low dissipative (underdamping) cases, $T^{*}$ is approximately given by ${ }^{66,67}$

$$
T^{*}=\frac{\hbar \hat{\omega}_{p}(y=\langle y\rangle)}{2 \pi k_{B}} .
$$

Here $\langle y\rangle=\int_{0}^{1} d y P(y) y$ is the average switching current, where $P(y)$ is the switching current distribution which is related to the escape rate $\Gamma$ as ${ }^{60}$

$$
P(y)=\frac{1}{v} \Gamma(y) \exp \left[-\frac{1}{v} \int_{0}^{y} \Gamma\left(y^{\prime}\right) d y^{\prime}\right] .
$$

In this equation, $v \equiv|d y / d t|$ is the sweep rate of the external bias current. Importantly, $T^{*}$ is reduced in the presence of dissipative effects. ${ }^{68}$

By using Eqs. (36) and (39), we calculate $\Gamma$ and compare it with the case without the quasiparticle dissipation. In Fig. 9, we numerically plot $\Gamma$ and $\Gamma_{0}$ for $C=800 \mathrm{pF}$ and $\left|I_{c}\right|=$ $500 \mu \mathrm{A}^{13}$ and several values of $R$, where $\Gamma_{0}$ is the MQT escape rate in the absence of the quasiparticle dissipation $\left[C_{\text {ren }} \rightarrow C\right.$ in Eq. (39)]. As seen in this figure, $\Gamma$ shows strong dependence on the junction resistance $R$, and $\Gamma$ is almost identical to $\Gamma_{0}$ in the case of large $R$, e.g., $R=55 \mathrm{~m} \Omega$ $(\gg \hbar / C \Delta \approx 0.63 \mathrm{~m} \Omega$ ), which corresponds to the actual SIFS junction. ${ }^{13}$ We also calculate $T^{*}$ for a realistic case $(R=55$ $\mathrm{m} \Omega$ ) and find that $T^{*}=7.4 \mathrm{mK}$ for the dissipative case $\left(C_{\text {ren }}=C+\delta C\right)$ and $T^{*}=7.5 \mathrm{mK}$ for the dissipationless

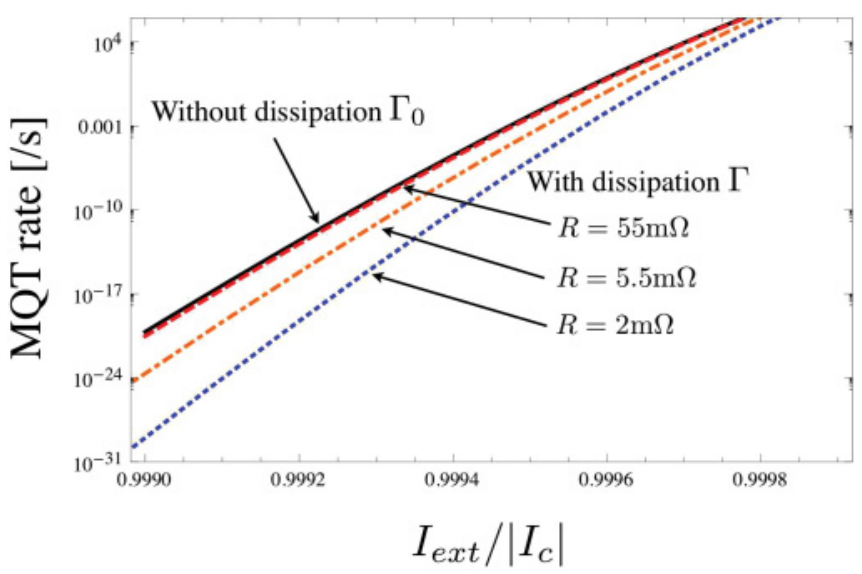

FIG. 9. (Color online) The MQT escape rate for a current-biased SIFS junction as a function of the bias current $I_{\text {ext }}$ for several values of the junction resistance $R . \Gamma_{0}$ (black solid line) and $\Gamma$ (red dashed, yellow dot-dashed, and blue dotted lines) are the MQT escape rate without and with quasiparticle dissipation, respectively. Parameters are $C=800 \mathrm{pF}, \Delta=1.3 \mathrm{meV}$, and $\left|I_{c}\right|=500 \mu \mathrm{A}$ (Ref. 13). case $\left(C_{\text {ren }}=C\right)$. As expected, the $T^{*}$ suppression is small enough to allow experimental observations of MQT. Thus we can conclude that the influence of the quasiparticle dissipation on the macroscopic quantum dynamics of SIFS junction is very small for the case when the condition Eq. (37) is hold. This fact strongly suggests the great advantage of realistic SIFS junctions for qubit applications. The smallness of the quasiparticle dissipation in SIFS junctions is due to the superconducting gap in the left $\mathrm{S}$ electrode and the strong insulating barrier $\left(\gamma_{B 1} \gg 1\right)$ between the left $S$ and $F$ layers.

It is important to note that such a weak quasiparticledissipation nature of MQT has been also predicted in $\pi$ junctions based on S/ferromagnetic insulator (FI)/S junctions. ${ }^{64,65}$ However no ferromagnetic insulator based Josephson junctions have been experimentally realized at present. On the other hands, the fabrication of SIFS junction is easily realized based on the current fabrication technology. ${ }^{13-15}$

\section{CONCLUSION}

We have developed a quantitative theory that describes the properties of the DOS and the current-voltage characteristics of a SIFS junction in the dirty limit. We considered the case of a strong insulating barrier in a SIFS junction such that the left $\mathrm{S}$ layer and the right $\mathrm{F} / \mathrm{S}$ bilayer are decoupled. In this case we can obtain the current-voltage characteristics of a SIFS junction in the framework of standard tunnel theory. In order to calculate quasiparticle current we first calculated the DOS in the ferromagnetic layer of the $\mathrm{F} / \mathrm{S}$ bilayer. We described the DOS behavior as a function of parameters characterizing properties of the ferromagnetic layer. In our theory we consider three such parameters: thickness of the ferromagnetic layer $d_{f}$, exchange field $h$, and magnetic scattering $\tau_{m}$. We have discussed the DOS properties paying special attention to the DOS oscillations at the Fermi energy. We have also proposed to measure the exchange field in experiments on weak ferromagnets by measuring the DOS peak at $E=h$. We compared the results, obtained with a self-consistent numerical method, with a known analytical DOS approximation, which is valid when the ferromagnetic layer is thick enough.

Using the numerically obtained DOS we have calculated the current-voltage characteristics of a SIFS junction and have observed features which are the signatures of the proximity effect in the $S / F$ bilayer. We showed that there exists typical shape patterns of current-voltage characteristics related to the typical DOS structures in the ferromagnetic interlayer.

Finally, we have calculated the macroscopic quantum tunneling escape rate for the current-biased SIFS junctions by taking into account the dissipative correction due to the quasiparticle tunneling. Based on this we concluded that the influence of the quasiparticle dissipation on the macroscopic quantum dynamics of SIFS junctions is small, which is a great advantage of SIFS junctions for qubit applications compared to other types of ferromagnetic $\pi$ junctions.

\section{ACKNOWLEDGMENTS}

The authors thank A. I. Buzdin, E. Goldobin, D. A. Ivanov, A. V. Vedyayev, and M. Weides for useful discussions. This work was supported by NanoSCIERA project Nanofridge, 
ANR DYCOSMA, RFBR Project No. N11-02-12065, JSTCREST, a Grant-in-Aid for Scientific Research from the Ministry of Education, Science, Sports, and Culture of Japan (Grant No. 22710096), the Invitation Program for Foreign Young Researchers in the G-COE program "Education and
Research Center for Material Innovation," and the Spanish MICINN (Contract No. FIS2008-04209). F.S.B. thanks Intramural Special Project (Ref. 2009601036). A.A.G. thanks Dutch FOM for support. A.S.V. acknowledge the hospitality of Nanomaterials theory group, AIST, during his stay in Japan.
${ }^{1}$ L. N. Bulaevskiǐ, V. V. Kuziľ, and A. A. Sobyanin, JETP Lett. 25, 290 (1977).

${ }^{2}$ V. V. Ryazanov, V. A. Oboznov, A. Yu. Rusanov, A. V. Veretennikov, A. A. Golubov, and J. Aarts, Phys. Rev. Lett. 86, 2427 (2001); V. V. Ryazanov, V. A. Oboznov, A. V. Veretennikov, and A. Yu. Rusanov, Phys. Rev. B 65, 020501(R) (2001).

${ }^{3}$ Y. Blum, A. Tsukernik, M. Karpovski, and A. Palevski, Phys. Rev. Lett. 89, 187004 (2002).

${ }^{4}$ H. Sellier, C. Baraduc, F. Lefloch, and R. Calemczuk, Phys. Rev. Lett. 92, 257005 (2004).

${ }^{5}$ A. Bauer, J. Bentner, M. Aprili, M. L. Della Rocca, M. Reinwald, W. Wegscheider, and C. Strunk, Phys. Rev. Lett. 92, 217001 (2004).

${ }^{6}$ C. Bell, R. Loloee, G. Burnell, and M. G. Blamire, Phys. Rev. B 71, 180501(R) (2005).

${ }^{7}$ V. Shelukhin, A. Tsukernik, M. Karpovski, Y. Blum, K. B. Efetov, A. F. Volkov, T. Champel, M. Eschrig, T. Lofwander, G. Schon, and A. Palevski, Phys. Rev. B 73, 174506 (2006).

${ }^{8}$ V. A. Oboznov, V. V. Bol'ginov, A. K. Feofanov, V. V. Ryazanov, and A. I. Buzdin, Phys. Rev. Lett. 96, 197003 (2006).

${ }^{9}$ T. Kontos, M. Aprili, J. Lesueur, F. Genet, B. Stephanidis, and R. Boursier, Phys. Rev. Lett. 89, 137007 (2002).

${ }^{10}$ W. Guichard, M. Aprili, O. Bourgeois, T. Kontos, J. Lesueur, and P. Gandit, Phys. Rev. Lett. 90, 167001 (2003).

${ }^{11}$ F. Born, M. Siegel, E. K. Hollmann, H. Braak, A. A. Golubov, D. Yu. Gusakova, and M. Yu. Kupriyanov, Phys. Rev. B 74, 140501(R) (2006).

${ }^{12}$ G. P. Pepe, R. Latempa, L. Parlato, A. Ruotolo, G. Ausanio, G. Peluso, A. Barone, A. A. Golubov, Ya. V. Fominov, and M. Yu. Kupriyanov, Phys. Rev. B 73, 054506 (2006).

${ }^{13}$ M. Weides, M. Kemmler, E. Goldobin, D. Koelle, R. Kleiner, H. Kohlstedt, and A. Buzdin, Appl. Phys. Lett. 89, 122511 (2006).

${ }^{14}$ M. Weides, M. Kemmler, H. Kohlstedt, R. Waser, D. Koelle, R. Kleiner, and E. Goldobin, Phys. Rev. Lett. 97, 247001 (2006); M. Weides, C. Schindler, and H. Kohlstedt, J. Appl. Phys. 101, 063902 (2007).

${ }^{15}$ M. Weides, Ph. D. thesis, Forschungszentrums Jülich, 2007.

${ }^{16} \mathrm{~J}$. Pfeiffer, M. Kemmler, D. Koelle, R. Kleiner, E. Goldobin, M. Weides, A. K. Feofanov, J. Lisenfeld, and A. V. Ustinov, Phys. Rev. B 77, 214506 (2008).

${ }^{17}$ A. A. Bannykh, J. Pfeiffer, V. S. Stolyarov, I. E. Batov, V. V. Ryazanov, and M. Weides, Phys. Rev. B 79, 054501 (2009).

${ }^{18}$ M. Kemmler, M. Weides, M. Weiler, M. Opel, S. T. B. Goennenwein, A. S. Vasenko, A. A. Golubov, H. Kohlstedt, D. Koelle, R. Kleiner, and E. Goldobin, Phys. Rev. B 81, 054522 (2010).

${ }^{19}$ M. S. Anwar, F. Czeschka, M. Hesselberth, M. Porcu, and J. Aarts, Phys. Rev. B 82, 100501(R) (2010).

${ }^{20}$ T. S. Khaire, M. A. Khasawneh, W. P. Pratt Jr., and N. O. Birge, Phys. Rev. Lett. 104, 137002 (2010).
${ }^{21}$ W. A. Robinson, J. D. S. Witt, and M. G. Blamire, Science 329, 59 (2010).

${ }^{22}$ A. I. Buzdin, Rev. Mod. Phys. 77, 935 (2005).

${ }^{23}$ A. A. Golubov, M. Yu. Kupriyanov, and E. Il'ichev, Rev. Mod. Phys. 76, 411 (2004).

${ }^{24}$ F. S. Bergeret, A. F. Volkov, and K. B. Efetov, Rev. Mod. Phys. 77, 1321 (2005).

${ }^{25}$ E. Terzioglu and M. R. Beasley, IEEE Trans. Appl. Supercond. 8, 48 (1998); L. B. Ioffe, V. B. Geshkenbein, M. V. Feigel'man, A. L. Fauchère, and G. Blatter, Nature (London) 398, 679 (1999); G. Blatter, V. B. Geshkenbein, and L. B. Ioffe, Phys. Rev. B 63, 174511 (2001); A. V. Ustinov and V. K. Kaplunenko, J. Appl. Phys. 94, 5405 (2003); T. Ortlepp, A. Ariando, O. Mielke, C. J. M. Verwijs, K. F. K. Foo, H. Rogalla, F. H. Uhlmann, and H. Hilgenkamp, Science 312, 1495 (2006).

${ }^{26}$ A. K. Feofanov, V. A. Oboznov, V. V. Bolginov, J. Lisenfeld, S. Poletto, V. V. Ryazanov, A. N. Rossolenko, M. Khabipov, D. Balashov, A. B. Zorin, P. N. Dmitriev, V. P. Koshelets, and A. V. Ustinov, Nature Phys. 6, 593 (2010).

${ }^{27}$ H. Hilgenkamp, Supercond. Sci. Technol. 21, 034011 (2008).

${ }^{28}$ A. Buzdin, Phys. Rev. Lett. 101, 107005 (2008); N. G. Pugach, E. Goldobin, R. Kleiner, and D. Koelle, Phys. Rev. B 81, 104513 (2010).

${ }^{29}$ A. S. Vasenko, A. A. Golubov, M. Yu. Kupriyanov, and M. Weides, Phys. Rev. B 77, 134507 (2008).

${ }^{30}$ N. G. Pugach, M. Yu. Kupriyanov, A. V. Vedyayev, C. Lacroix, E. Goldobin, D. Koelle, R. Kleiner, and A. S. Sidorenko, Phys. Rev. B 80, 134516 (2009).

${ }^{31}$ A. F. Volkov and K. B. Efetov, Phys. Rev. Lett. 103, 037003 (2009).

${ }^{32}$ M. Yu. Kuprianov and V. F. Lukichev, Zh. Eksp. Teor. Fiz. 94, 139 (1988) [Sov. Phys. JETP 67, 1163 (1988)].

${ }^{33}$ E. V. Bezuglyi, A. S. Vasenko, V. S. Shumeiko, and G. Wendin, Phys. Rev. B 72, 014501 (2005); E. V. Bezuglyi, A. S. Vasenko, E. N. Bratus, V. S. Shumeiko, and G. Wendin, ibid. 73, 220506(R) (2006).

${ }^{34}$ N. R. Werthamer, Phys. Rev. 147, 255 (1966).

${ }^{35}$ K. D. Usadel, Phys. Rev. Lett. 25, 507 (1970).

${ }^{36}$ E. A. Demler, G. B. Arnold, and M. R. Beasley, Phys. Rev. B 55, 15174 (1997).

${ }^{37}$ D. Yu. Gusakova, A. A. Golubov, M. Yu. Kupriyanov, and A. Buzdin, Pis'ma Zh. Eksp. Teor. Fiz. 83, 385 (2006) [JETP Lett. 83, 327 (2006)].

${ }^{38}$ M. Faure, A. I. Buzdin, A. A. Golubov, and M. Yu. Kupriyanov, Phys. Rev. B 73, 064505 (2006).

${ }^{39}$ F. S. Bergeret, A. F. Volkov, and K. B. Efetov, Phys. Rev. B 75, 184510 (2007)

${ }^{40}$ D. A. Ivanov, Ya. V. Fominov, M. A. Skvortsov, and P. M. Ostrovsky, Phys. Rev. B 80, 134501 (2009). 
${ }^{41}$ A. A. Abrikosov and L. P. Gor'kov, Zh. Eksp. Teor. Fiz. 39, 1781 (1960) [Sov. Phys. JETP 12, 1243 (1961)].

${ }^{42}$ D. Huertas-Hernando, Yu. V. Nazarov, and W. Belzig, Phys. Rev. Lett. 88, 047003 (2002); A. Cottet and W. Belzig, Phys. Rev. B 72, 180503(R) (2005); A. Cottet, D. Huertas-Hernando, W. Belzig, and Y. V. Nazarov, ibid. 80, 184511 (2009).

${ }^{43}$ A. Cottet, Phys. Rev. B 76, 224505 (2007).

${ }^{44}$ A. Cottet and J. Linder, Phys. Rev. B 79, 054518 (2009).

${ }^{45}$ A. A. Golubov, M. Yu. Kupriyanov, and Ya. V. Fominov, Pis'ma Zh. Eksp. Teor. Fiz. 75, 223 (2002) [JETP Lett. 75, 190 (2002)].

${ }^{46}$ A. A. Golubov and M. Yu. Kupriyanov, J. Low Temp. Phys. 70, 83 (1988); Zh. Eksp. Teor. Fiz. 96, 1420 (1989) [Sov. Phys. JETP 69, 805 (1989)].

${ }^{47}$ A. A. Golubov, E. P. Houwman, J. G. Gijsbertsen, V. M. Krasnov, J. Flokstra, H. Rogalla, and M. Yu. Kupriyanov, Phys. Rev. B 51, 1073 (1995).

${ }^{48}$ L. Cretinon, A. K. Gupta, H. Sellier, F. Lefloch, M. Faure, A. Buzdin, and H. Courtois, Phys. Rev. B 72, 024511 (2005).

${ }^{49}$ R. Fazio and C. Lucheroni, Europhys. Lett. 45, 707 (1999); D. A. Ivanov and Ya. V. Fominov, Phys. Rev. B 73, 214524 (2006).

${ }^{50}$ B. Crouzy, E. Bascones, and D. A. Ivanov, Phys. Rev. B 72, 092501 (2005).

${ }^{51}$ T. Kontos, M. Aprili, J. Lesueur, and X. Grison, Phys. Rev. Lett. 86, 304 (2001).

${ }^{52}$ A. Buzdin, Phys. Rev. B 62, 11377 (2000).

${ }^{53}$ T. Yokoyama, Y. Tanaka, and A. A. Golubov, Phys. Rev. B 72, 052512 (2005).
${ }^{54}$ F. Zhou, P. Charlat, B. Spivak, and B. Pannetier, J. Low Temp. Phys. 110, 841 (1998).

${ }^{55}$ T. Kontos, M. Aprili, J. Lesueur, X. Grison, and L. Dumoulin, Phys. Rev. Lett. 93, 137001 (2004)

${ }^{56}$ E. Goldobin (private communication).

${ }^{57}$ P. SanGiorgio, S. Reymond, M. R. Beasley, J. H. Kwon, and K. Char, Phys. Rev. Lett. 100, 237002 (2008).

${ }^{58}$ I. Petkovic and M. Aprili, Phys. Rev. Lett. 102, 157003 (2009); V. M. Krasnov, T. Golod, T. Bauch, and P. Delsing, Phys. Rev. B 76, 224517 (2007).

${ }^{59}$ J. M. Martinis, S. Nam, J. Aumentado, and C. Urbina, Phys. Rev. Lett. 89, 117901 (2002).

${ }^{60}$ A. Wallraff, A. Lukashenko, C. Coqui, A. Kemp, T. Duty, and A. V. Ustinov, Rev. Sci. Instrum. 74, 3740 (2003).

${ }^{61}$ G. Schön and A. D. Zaikin, Phys. Rep. 198, 237 (1990).

${ }^{62}$ V. Ambegaokar, U. Eckern, and G. Schön, Phys. Rev. Lett. 48, 1745 (1982).

${ }^{63}$ S. Kawabata, S. Kashiwaya, Y. Asano, and Y. Tanaka, Phys. Rev. B 70, 132505 (2004); 72, 052506 (2005).

${ }^{64}$ S. Kawabata, S. Kashiwaya, Y. Asano, Y. Tanaka, and A. A Golubov, Phys. Rev. B 74, 180502(R) (2006).

${ }^{65}$ S. Kawabata, Y. Asano, Y. Tanaka, A. A. Golubov, and S. Kashiwaya, Phys. Rev. Lett. 104, 117002 (2010); S. Kawabata and Y. Asano, Low Temp. Phys. 36, 915 (2010).

${ }^{66} \mathrm{U}$. Weiss, Quantum Dissipative Systems (World Scientific, Singapore, 1999).

${ }^{67}$ T. Kato and M. Imada, J. Phys. Soc. Jpn. 65, 2963 (1996).

${ }^{68}$ A. O. Caldeira and A. J. Leggett, Ann. Phys. (NY) 149, 374 (1983). 